\title{
IBS Simulation with different RF configurations in RHIC
}

\author{
C. Liu, A. Fedotov, M. Minty, V. Ptitsyn
}

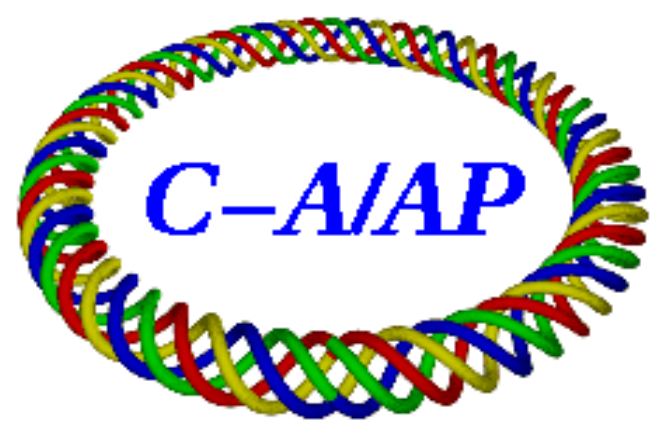

\section{Collider-Accelerator Department Brookhaven National Laboratory Upton, NY 11973}

\author{
U.S. Department of Energy \\ Office of Science, Office of Nuclear Physics
}

Notice: This document has been authorized by employees of Brookhaven Science Associates, LLC under Contract No. DE-SC0012704 with the U.S. Department of Energy. The United States Government retains a nonexclusive, paid-up, irrevocable, world-wide license to publish or reproduce the published form of this document, or allow others to do so, for United States Government purposes. 


\title{
IBS SIMULATION WITH DIFFERENT RF CONFIGURATIONS IN RHIC
}

\author{
C. Liu, A. Fedotov, M. Minty, V. Ptitsyn, Collider Accelerator Department, Brookhaven \\ National Lab, Upton, NY, USA.
}

Abstract

It is a crucial task to understand the beam emittance growth during RHIC cycle and the underlying causes. One would benefit not just for the current operation of RHIC, also for the design of eRHIC. This report focuses on the Intra-Beam Scattering (IBS) contribution to the emittance growth of the proton beam with two different configurations of RF system. The answers to these questions will be given in the end of the report; can IBS explain the emittance growth all alone? What's the difference of IBS growth rates for different RF configurations?

\section{IBS OVERVIEW}

The IBS are small angle coulomb scattering between particles, which would cause beam dimensions in phase space to grow. Many theories of IBS have been developed in the past [1-5]. When beam energy is below transition energy, an equilibrium beam distribution exists so that particles only exchange energy between transverse and longitudinal dimensions. With energy above transition, beam dimensions expand simultaneously. The energy of proton beam (24 GeV) at injection in RHIC is above transition energy. One could use the high energy approximation as guidance and here we quote the rates expressed in reference [4]:

$$
\left[\begin{array}{c}
\frac{1}{\sigma_{p}} \frac{d \sigma_{p}}{d t} \\
\frac{1}{\sigma_{x}} \frac{d \sigma_{x}}{d t} \\
\frac{1}{\sigma_{y}} \frac{d \sigma_{y}}{d t}
\end{array}\right]=\frac{A_{0}}{2} \int e^{-D_{z}} \ln \left(1+C^{4} z^{2}\right)\left[\begin{array}{c}
n_{b}\left(1-d^{2}\right) g_{1} \\
a^{2} g_{2}+\left(d^{2}+\bar{d}^{2}\right) g_{1} \\
b^{2} g_{3}
\end{array}\right] \sin \theta d \theta d \phi d z
$$

where $A_{0}=\frac{c r_{0}^{2} N Z^{4} \beta_{x} \beta_{y}}{32 \pi^{2} A^{2} \sigma_{x}^{2} \sigma_{y}^{2} \sigma_{p} \sigma_{s} \beta^{3} \gamma^{4}}, d=\frac{D_{p} \sigma_{p}}{\left(\sigma_{x}^{2}+D_{p}^{2} \sigma_{p}^{2}\right)^{1 / 2}}, \bar{d}=\frac{\bar{D}_{p} d}{D_{p}}, \bar{D}_{p}=\alpha_{x} D_{p}+\beta_{x} D_{p}^{\prime}, a=\frac{\beta_{x} d}{D_{p} \gamma}, b=\frac{\beta_{y} \sigma_{x}}{\beta_{x} \sigma_{y}} a, D=$ $\cos ^{2} \theta+b^{2} \sin ^{2} \theta \sin ^{2} \phi+(a \sin \theta \cos \phi-\bar{d} \cos \theta)^{2}, C=2 \beta \sigma_{p}\left[\sigma_{y}\left(1-d^{2}\right) / r_{0}\right]^{1 / 2}, g_{1}=1-3 \cos ^{2} \theta, g_{2}=$ $\cos ^{2} \theta-2 \sin ^{2} \theta \cos ^{2} \phi+\sin ^{2} \theta \sin ^{2} \phi+6 \bar{d} \cos \theta \sin \theta \cos \phi / a, g_{3}=\cos ^{2} \theta+\sin ^{2} \theta \cos ^{2} \phi-2 \sin ^{2} \theta \sin ^{2} \phi$. The growth rates increase with the beam phase space density, charge number, and decrease with beam energy. In addition, the energy spread itself also effect the growth rates.

\section{SIMULATION CODE AND INPUTS}

The simulation was performed with code BETACOOL [6]. Of many simulation functions provided by BETACOOL, only IBS calculation is performed for our case.

The accelerating RF in RHIC was switched from $28 \mathrm{MHz}$ to $9 \mathrm{MHz}$ cavity in 2010 for better matching of the longitudinal emittance of the incoming beam from AGS and the RF bucket in RHIC. For eRHIC, the ring-ring design requires 360 ion bunches. Therefore, the ion beam needs to be accelerated by the $28 \mathrm{MHz}$ cavities and then hopefully can be re-bucketed in higher harmonic cavities. Under this circumstance, it is interesting to compare the IBS growth rates and emittance evolution with these two RF configurations. 
The particle distribution in 3D space are assumed Gaussian. The transverse emittance was adjusted to match the values measured by the IPMs (Ionization Profile Monitors). The longitudinal energy spread is needed with the RF configurations as inputs to define the longitudinal emittance. The RF configuration was set according to the operational conditions. The beam energy spread is given by [7]

$$
\frac{\sigma_{p}}{p}=\left(-\frac{h e V \cos \phi_{s} S^{2} f_{0}^{2}}{18 \pi \eta E_{s}^{3} \beta^{2}}\right)^{1 / 4}
$$

where $h$ is the harmonic number, $V$ is the cavity voltage, $\phi_{s}$ is the synchronous phase, $S$ is the $95 \%$ longitudinal emittance, $f_{0}$ is the revolution frequency, $\eta$ is the slip factor, $E_{s}$ is the beam energy, $\beta$ is the Lorentz factor.

The 95\% longitudinal emittance is estimated to be $\sim 1 \mathrm{eV} \cdot s$ from AGS. With $9 \mathrm{MHz}$ cavity at $19 \mathrm{kV}, \frac{\sigma_{p}}{p}=$ $\left(\frac{120 * 19000 * 1^{2} *\left(78 * 10^{4}\right)^{2}}{18 \pi * 4.4 * 10^{-4} *\left(24 * 10^{9}\right)^{3}}\right)^{\frac{1}{4}}=4.5 * 10^{-4}$. With $28 \mathrm{MHz}$ cavity at $120 \mathrm{kV}, \frac{\sigma_{p}}{p}=\left(\frac{360 * 120000 * 1^{2} *\left(78 * 10^{4}\right)^{2}}{18 \pi * 4.4 * 10^{-4} *\left(24 * 10^{9}\right)^{3}}\right)^{\frac{1}{4}}=9.3 *$ $10^{-4}$.

The Martini model was chosen for the IBS rates calculation. The integral can be done numerically first, then one can adjust the coulomb logarithm to match the previously calculated growth rates. Thereafter, the fast calculation with coulomb logarithm can be done for the emittance evolution calculation. The Mad-X Twiss output of pp15-e0 lattice [8] was imported in BETACOOL for simulation.

\section{SIMULATION VS MEASUREMENT}

For proton beam at injection with $9 \mathrm{MHz}$, the settings of the BETACOOL simulation are shown below in Fig. 1. RHIC was assumed as a well-decoupled machine in this case of simulation. The un-normalized transverse emittances were adjusted so that the normalized 95\% emittances match with the measured emittances by IPMs shown in Fig. 5. The energy spread was also adjusted started from the theoretical value from the previous section so that the bunch length matches the rms value measured by Wall Current Monitor (WCM) [9]. The $28 \mathrm{MHz}$ cavity was on with $100 \mathrm{kV}$ voltage for Landau damping. The measurement precision of IPMs has been improved over the years. Therefore, measurement data was chosen from $100 \mathrm{GeV}$ polarized proton run in 2015 for comparison with simulations.
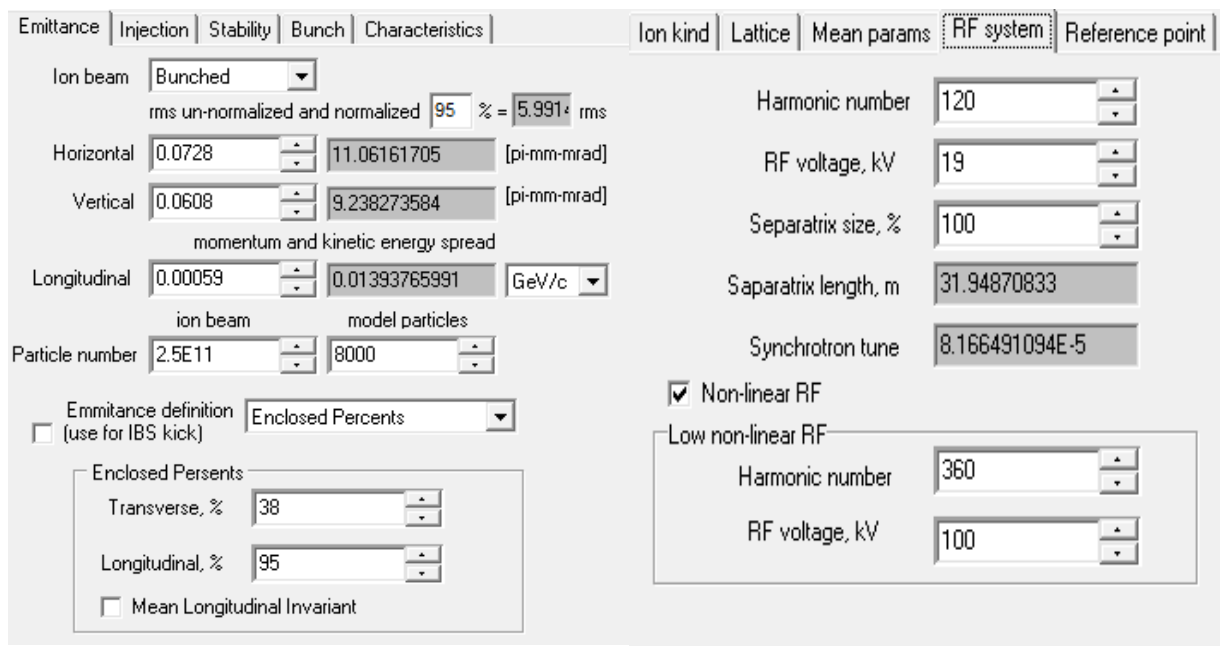

Fig. 1 Inputs for BETACOOL program for the case with $9 \mathrm{MHz}$ RF system, includes beam emittance in three dimensions and RF configuration. 
From the simulation, the horizontal growth rate is $3.35 \mathrm{E}-5[1 / \mathrm{sec}]$, the vertical growth rate is $-3.48 \mathrm{E}-6[1 / \mathrm{sec}]$, and the longitudinal growth rate is $1.47 \mathrm{E}-4[1 / \mathrm{sec}]$ for the decoupled case.

The longitudinal bunch length evolution from simulation and measurement are shown both in Fig. 2 for comparison. The relative difference of bunch length between measurement and simulation is about $4 \%$ in half an hour.

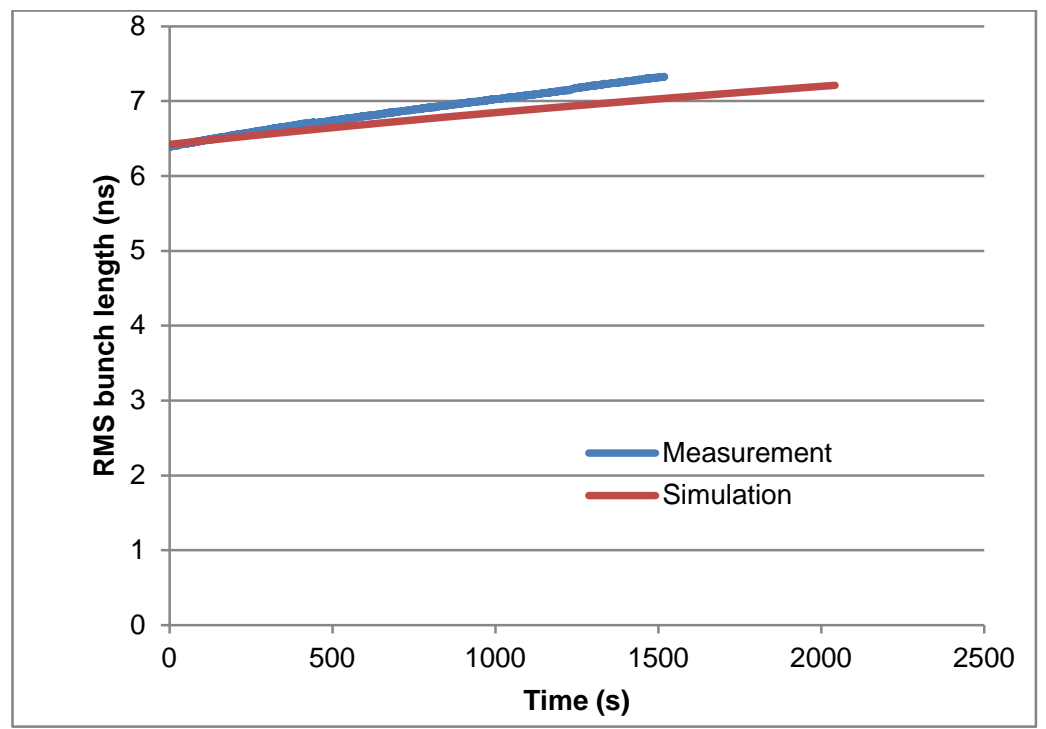

Fig. 2 Comparison between measured (fill \# 18899) and simulated bunch length evolution over 30 minutes.

The transverse emittance evolution is shown below.

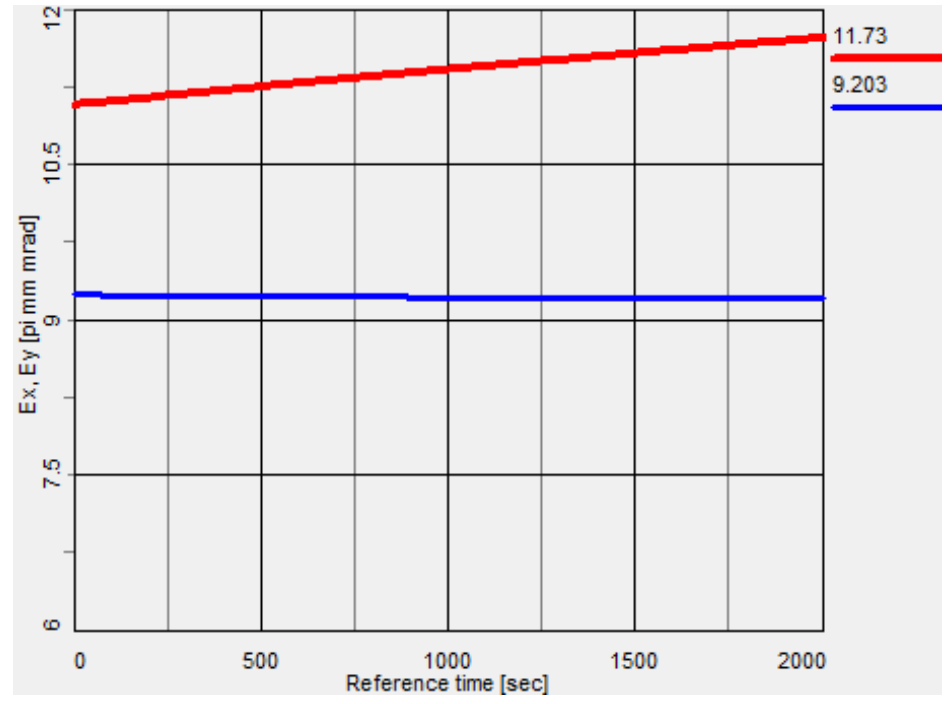

Fig. 3 Transverse emittance evolution simulated by BETACOOL for the decoupled case with the $9 \mathrm{MHz}$ RF system; the horizontal emittance is in red and vertical emittance in blue. 
If one assumes full coupling between horizontal and vertical planes in the simulation, the horizontal growth rate is $1.53 \mathrm{E}-5[1 / \mathrm{sec}]$, the vertical growth rate is $1.83 \mathrm{E}-5[1 / \mathrm{sec}]$, and the longitudinal growth rate is $1.47 \mathrm{E}-4[1 / \mathrm{sec}]$. With full coupled machine, the transverse growth rates are average of the rates in both planes in the case of no coupling. The evolution of transverse emittances is shown in below. The evolutions of bunch length are the same for coupled and decoupled cases.

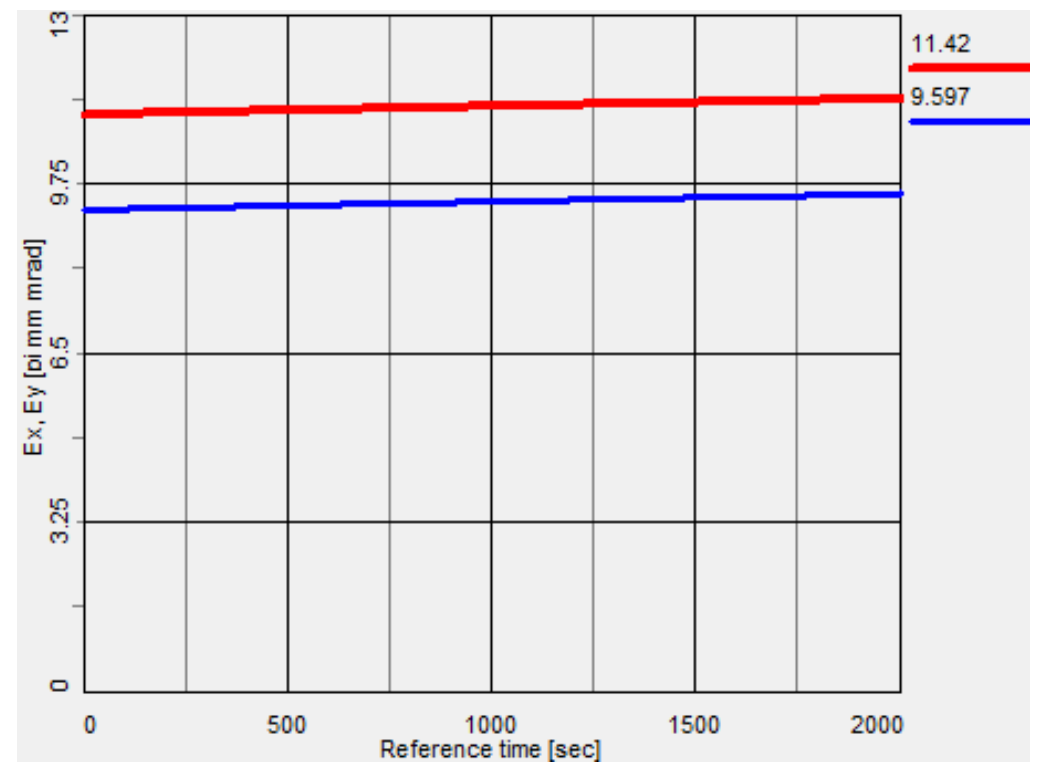

Fig. 4 Transverse emittance evolution simulated by BETACOOL for the coupled case with $9 \mathrm{MHz}$ RF system

The proton beams were sitting at injection about half an hour during the fill 18899, Run-15 $100 \mathrm{GeV}$ polarized proton program. The measured transverse emittances by IPMs are shown below in the upper plot of Figure 5, with the fit error in the lower plot. The IPM profile measurement and corresponding Gaussian fit are in a good agreement with a fit error around and below 0.1 as shown. In both coupled and decoupled cases, we observed some discrepancy of transverse emittance evolution between simulation and measurement.
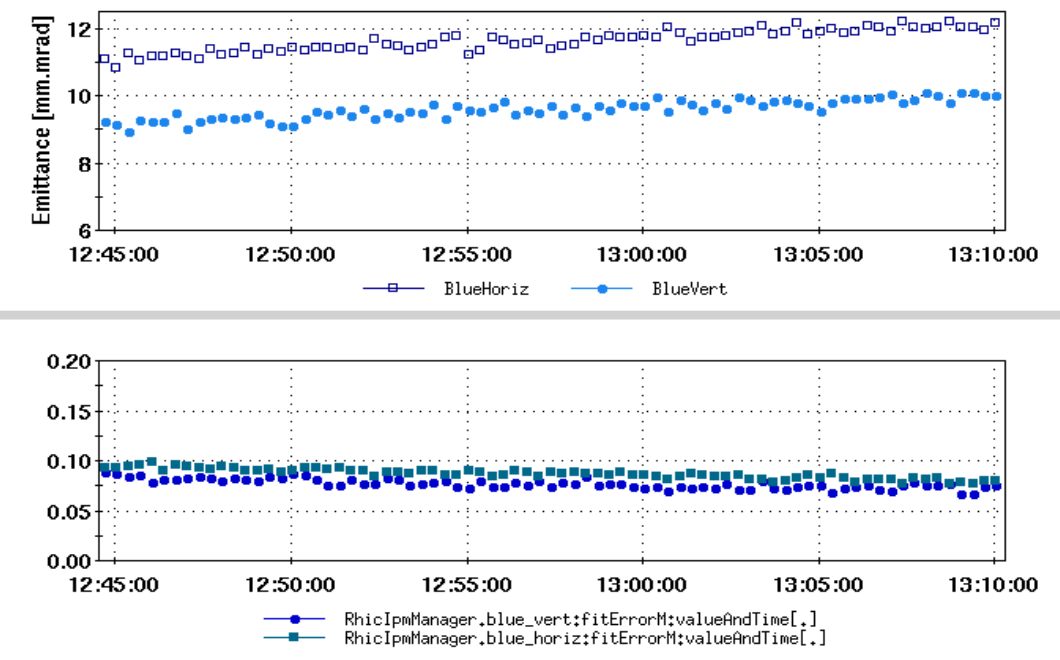

Fig. 5 Transverse emittances measured by IPMs shown in upper plot and the fit error in the lower plot, during fill 18899. 


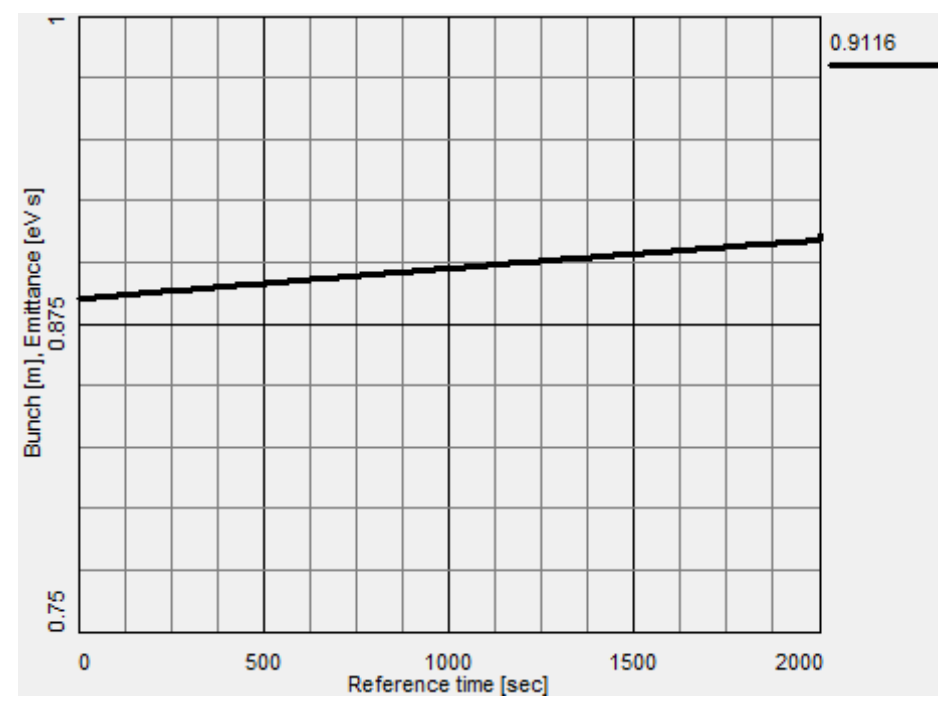

Fig. 5 The bunch length evolution simulated by BETACOOL for the coupled case with $28 \mathrm{MHz}$ RF system

From the simulation, the horizontal growth rate is $2.85 \mathrm{E}-5[1 / \mathrm{sec}]$, the vertical growth rate is $3.42 \mathrm{E}-5[1 / \mathrm{sec}]$, and the longitudinal growth rate is $2.89 \mathrm{E}-5[1 / \mathrm{sec}$. Compared with the growth rates with $9 \mathrm{MHz} \mathrm{RF}$ system coupled case, the transverse rates with $28 \mathrm{MHz}$ RF system are higher while the longitudinal rate is lower.

\section{DISCUSSION}

The agreement between simulated and measured emittance with IBS is worse for proton beam than for gold beam [10]. The growth rate is proportional to $\frac{N Z^{4}}{A^{2}}$, therefore the rates for gold beam is about 10 times higher than that of proton beam. With comparable noise contribution to the emittance evolution, IBS contribution is dominating for $\mathrm{Au}$ beam, but not for proton beam.

Compared with previous efforts [11,12] to bench-mark the simulation with measurements, the measurements of transverse emittance and bunch length are more robust and precise.

Experimental studies [13] on beam emittance in RHIC has been carried out in the past and will resume in the future.

\section{SUMMARY}

With $9 \mathrm{MHz}$ cavity, the simulated longitudinal growth rate is higher, the transverse growth rate is lower than those with $28 \mathrm{MHz}$ cavity due to the smaller energy spread. The coupling of RHIC is visible from the evolution of transverse emittance measured by IPMs. The growth rates from simulation are generally lower than those from measurements, especially so in transverse planes.

\section{REFERENCES}

[1] A. Piwinski, CERN Acc. School (1991) p.126

[2] J.D. Bjorken, S.K. Mtingwa, Part. Accel. 13(1983) 115 
[3] M. Martini, CERN PS/84-9(AA) 1984

[4] G. Parsen, NIM A256 (1987) 231

[5] J. Wei, PAC 93, p.3651

[6] I. Meshkov et al., BETACOOL code, JINR Interim report. 2002

[7] D. A. Edwards and M. J. Syphers, An Introduction to the Physics of High Energy Accelerators, J. Wiley, \& Sons, Inc., New York (1993).

[8] G. Robert-Demolaize, Private Communication.

[9] K. Mernick, Private Communication.

[10] M. Blaskiewicz, Private Communication.

[11] W. Fisher, et al., EPAC’02, p236-238, Paris, France, 2002.

[12] A.V. Fedotov , W. Fischer, S. Tepikian, and J. Wei, Proceedings of HB2006, p259-261, Tsukuba, Japan.

[13] M. Minty, H. Huang, Private Communication. 